\title{
The Clinical Characteristics of Acute Pancreatitis in Gerontal Patients: A Retrospective Study
}

This article was published in the following Dove Press journal:

Clinical Interventions in Aging

\section{Bingjun Yu \\ Nianshuang Li \\ Jiarong $\mathrm{Li}$ \\ Jianhua Wan \\ Wenhua He \\ Yin Zhu \\ Nonghua Lu (D)}

Department of Gastroenterology, The First Affiliated Hospital of Nanchang University, Nanchang 330006, Jiangxi Province, People's Republic of China
Correspondence: Nonghua Lu Department of Gastroenterology, The First Affiliated Hospital of Nanchang University, 17 Yong Waizheng Street, Donghu District, Nanchang 330006, Jiangxi Province, People's Republic of China

Email lunonghua@ncu.edu.cn
Background: The incidence of acute pancreatitis (AP) in ageing patients has increased in recent years, and results regarding the clinical outcomes of these patients are controversial. The aim of this study was to compare the clinical outcomes of AP in ageing patients over 60 years old.

Methods: Eighty patients aged $\geq 80$ years (oldest group) were compared to 393 patients aged 60 to 79 years (older group). The clinical course and biochemical and radiological data were evaluated. The primary endpoints were mortality rate, intensive care unit (ICU) admission rate and in-hospital length of stay (LOS). The secondary endpoints were the incidence of operative treatment and complications of AP.

Results: Abdominal pain $(61.3 \%$ vs $46.3 \%, \mathrm{P}=0.013)$ was less common in the oldest group. Jaundice $(17.5 \%$ vs $8.9 \%, \mathrm{P}=0.021)$ and dyspnoea $(26.3 \%$ vs $11.5 \%, \mathrm{P}=0.001)$ were more obvious in the oldest group than in the older group. The mean BMI was lower in the oldest group than in the older group $(21.07 \pm 3.18$ vs $22.36 \pm 2.89, \mathrm{P}=0.001)$. Age over 80 years $(\mathrm{P}=0.011)$ and organ failure $(\mathrm{P}<0.05)$ were independent risk factors for mortality. More severe AP $(\mathrm{P}=0.001)$, abdominal pain $(\mathrm{P}=0.033)$ and organ failure $(\mathrm{P}<0.05)$ were associated with the ICU admission rate. Age over 80 years $(\mathrm{P}=0.001)$, more severe AP $(\mathrm{P}=0.001)$, female sex $(\mathrm{P}=0.018)$, jaundice $(\mathrm{P}=0.038)$, operative treatment $(\mathrm{P}<0.05)$ and organ failure $(\mathrm{P}<0.05)$ were risk factors for increased LOS.

Conclusion: The oldest group had a higher death rate and longer LOS than the older group. More attention should be given to the clinical symptoms of this frail population. We propose that more comprehensive and goal-directed attendant diagnostic procedures should be performed to detect the disease early and to improve the outcomes of ageing patients.

Keywords: acute pancreatitis, ageing patients, clinical traits, death rate, ICU admission rate

\section{Background}

In Europe over the past decade, improvements in medical care have resulted in a $2.4 \%$ increase in the number of individuals aged over 65 years. More importantly, it has been predicted that those over 80 years old are expected to comprise $12.7 \%$ of the population in 2080 , compared to $5.5 \%$ in $2007 .{ }^{1}$ With the ageing of the world population, trends in the intensification of ageing phenomena have become a major issue, and an increasing number of ageing patients have begun to experience acute pancreatitis (AP). ${ }^{2-4}$

AP is a potentially fatal clinical disease that is characterized by oedema, haemorrhage and necrosis. ${ }^{5-7}$ The clinical process of AP varies from self-limiting acute inflammation to a clinically severe condition, potentially leading to lifethreatening disease due to the risk of multi-organ failure. ${ }^{8}$ The mortality rate of 
AP varies extremely widely, from $5 \%$ for mild acute pancreatitis (MAP) $)^{9,10}$ to $30 \%$ for severe acute pancreatitis (SAP). ${ }^{11,12}$

Most studies have shown that AP is a life-threatening disease. After AP onset, frail elderly adults develop severe conditions more easily than younger patients do. Hence, it is necessary to determine the outcomes and clinical traits of AP in this growing population and recognize that useful medical and interventional treatment is crucial for them.

It is more difficult for physicians to diagnose AP in ageing patients compared to younger patients. The symptoms of ageing patients manifest later of the disease is characterized by a nonspecific presentation. ${ }^{13}$ Furthermore, the high comorbidity rate in the elderly population may result in severe consequences in AP. ${ }^{14-17}$

Although age is an element that influences the progression of $\mathrm{AP},{ }^{17-20}$ its influence on clinical traits remains controversial. The reason is probably because few studies have surveyed the clinical traits of AP in the ageing population. One study reported that ageing patients have a more severe course but do not have an increased mortality rate. ${ }^{14}$ Conversely, other studies have proposed that age itself is an independent risk factor for intensive care unit (ICU) admission and mortality. ${ }^{19,21}$ Overall, age is a key factor utilized to stratify the severity of the disease. ${ }^{8}$

These inconsistent findings may be due to the different age groupings used in different countries and by different authors, which makes it difficult to compare the clinical outcomes of AP in ageing patients. Hence, it is necessary to divide ageing patients into a heterogeneous cohort and then analyse the clinical outcomes of AP according to the different age subsets.

In our research, we focus our attention on subgroups of oldest patients ( $\geq 80$ years) and older patients (60-79 years) according to Chinese age standardization. The aim of our study was to investigate the clinical traits of AP in ageing patients in Asian.

\section{Methods}

\section{Patient Data}

This retrospective study enrolled all patients with AP from the AP database of the First Affiliated Hospital of Nanchang University and received approval from the ethics committee (database approval number: 2011001). The study comprised 2148 patients recruited from the AP database from January 1, 2018, to December 31, 2019. AP patients younger than 60 years were excluded. The total population was divided into two groups: the older group and the oldest group. The older group consisted of patients aged 60 to 80 years old, and the oldest group comprised AP patients aged over 80 years. We ultimately enrolled 473 ageing patients with AP in our study. The exclusion criteria are listed in Figure 1. Clinical data such as age, sex, history of smoking and drinking, diabetes, hypertension, history of renal diseases, complications, severity and aetiology of AP during hospitalization, death, results of

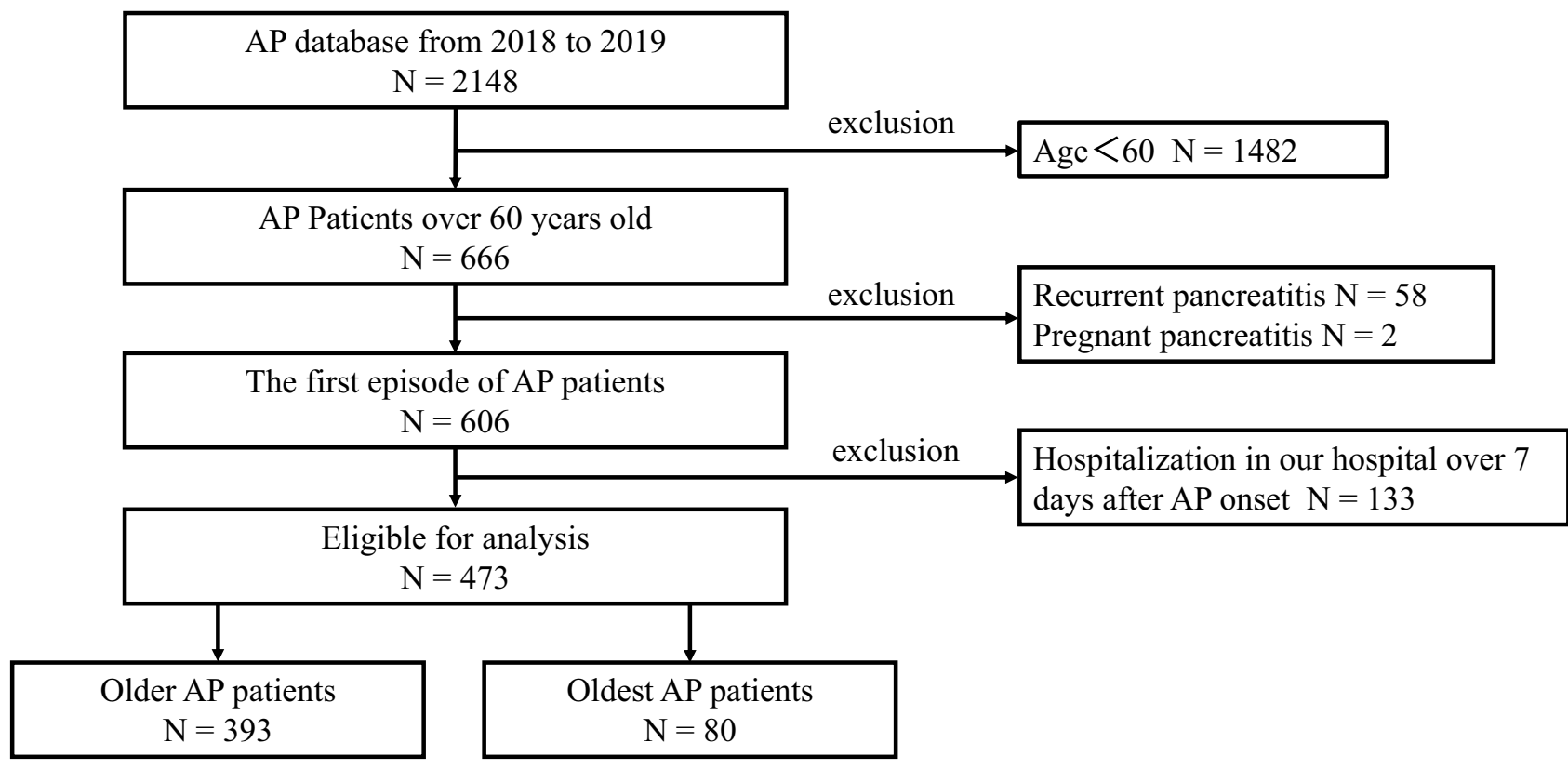

Figure I Flow chart of study participants. 
clinical tests for biomarkers, length of hospital stay after AP onset, ICU admission and body mass index (BMI) on admission were recorded. BMI $\left(\mathrm{kg} / \mathrm{m}^{2}\right)$ was defined as weight in kilograms divided by height in square metres. The Acute Physiology and Chronic Health Evaluation II (APACHE II) score, bedside index for severity of AP and the Marshall and Glasgow score were calculated based on patient data.

\section{Diagnosis and Definition}

The diagnosis of AP was based on the 2012 revised Atlanta classification criteria. $^{22}$ The 2012 revision of the Atlanta Acute Pancreatitis Diagnostic and Classification Criteria was used to classify AP severity and aetiology. Organ failure was assessed according to the modified Marshall scoring system. ${ }^{22}$ Pancreatic complications were diagnosed by contrast-enhanced computed tomography (CECT), which indicated diffuse enlargement of the pancreatic or peripancreatic volume, blurred edges, mild enhancement of the oedematous area after enhanced scanning, and no enhancement of necrotic areas. ${ }^{10,23}$ Death was regarded as death during hospitalization. Definitions are shown in Supplementary Table 1. Physical examination aimed to assess each patient's general health condition. The actual clinical course and the results of radiological (CT or CECT) and laboratory tests (white blood cells (WBC), lactic dehydrogenase $(\mathrm{LDH})$, alanine aminotransferase (ALT), aspartate transaminase (AST), total bilirubin (TBIL), direct bilirubin (DBIL), C-reactive protein (CRP), blood urea nitrogen (BUN), glucose (GLU), total cholesterol (TC), triglycerides (TG), albumin (ALB), amylase (AMY), and creatinine $(\mathrm{Cr})$ ) were assessed for each group. Those with chronic pancreatitis or pancreatic cancer were excluded from further assessment.

\section{Statistical Analysis}

Categorical variables were statistically compared by the chi-square test. The Mann-Whitney $U$-test was used for continuous variables. Categorical variables are presented as numbers and percentages, and continuous variables are presented as medians and mean differences; 0.05 was regarded as the significance level. Variables with significance in the univariate and multivariate analyses of the logistic regression model were used to determine the independent factors related to mortality, in-hospital length of stay (LOS) and ICU admission rate. Variables with a $\mathrm{P}$ value $<0.05$ in the univariate analysis were entered into a multivariable logistic regression to identify independent predictors. The results are expressed as odds ratios (ORs) with 95\% confidence intervals (CIs). All data were analysed by SPSS $24 \mathrm{R}$.

\section{Results}

From January 2018 to December 2019, a total of 2148 patients were admitted to the tertiary hospital of the First Affiliated Hospital of Nanchang University. Four hundred seventy-three ageing AP patients were included after excluding those that did not meet the study criteria. The flow chart is listed in Figure 1. The ageing AP patients in this cohort were divided into two groups: 393 patients in the older group, aged 60 to 80 years old, and 80 patients in the oldest group, aged $\geq 80$ years old. There were 217 males (45.9\%) and 256 females (54.1\%). The mean age was $70.46 \pm 7.67$ years for all AP patients. The LOS of the oldest group $(15.3 \pm 11.0)$ was longer than that of the older group (11.51 \pm 10.19$)$. For ICU admissions, there were 129 $(32.8 \%)$ patients in the older group and $30(37.5 \%)$ in the oldest group. The mean BMI of the oldest group (21.07 $\pm 3.18)$ was less than that of the older group (22.4 \pm 2.9$)$ $(\mathrm{P}=0.001)$. The detailed data are provided in Table 1 . The APACHE II score of the oldest group (13.08 \pm 3.50$)$ was higher than that of the older group $(11.9 \pm 3.7)(\mathrm{P}=0.01)$. Other scores are also listed in Table 2. History of smoking and drinking, chronic renal failure, hypertension, diabetes, severity of AP and incidence of complications are presented in Table 1 as baseline characteristics. However, there were no differences between the older group and oldest group with regard to biochemical values, including WBCs, serum amylase, and C-reactive protein (CRP), as shown in Table 2.

Various clinical symptoms were present in the AP patients. Abdominal pain occurred more frequently in the older group than in the oldest group (61.3\% vs $46.3 \%)$. In addition, symptoms of jaundice and dyspnoea more often occurred in the oldest AP patients, whereas the incidence of vomiting was similar between the older and oldest groups (6.6\% vs 8 10.0\%). In general, diarrhoea, jaundice, and dyspnoea were always accompanied by abdominal pain in the ageing AP patients. The details are listed in Table 1.

The main aetiology of AP among the ageing patients was biliary $(81.2 \%)$. A similar incidence of gallstones as the aetiology of AP was verified between the oldest group (88.6\%) and older group (79.6\%). Alcohol, hypertriglyceridemia, and other causes of AP are also indicated in Table 1. 
Table I Clinical Characteristics of AP Patients in the Older Group and Oldest Group

\begin{tabular}{|c|c|c|c|c|}
\hline & All Patients $(n=473)$ & Older Group (60-80 Years) $(n=393)$ & Oldest Group ( $\geq 80$ years) $(n=80)$ & $P$ \\
\hline Age (years) & $70.5 \pm 7.7$ & $67.8 \pm 5.3$ & $83.5 \pm 2.8$ & 0.001 \\
\hline \multicolumn{5}{|l|}{ Sex } \\
\hline Male & $217(45.9 \%)$ & $180(45.8 \%)$ & $37(46.3 \%)$ & 0.942 \\
\hline Female & $256(54.1 \%)$ & $213(54.2 \%)$ & $43(53.8 \%)$ & \\
\hline BMI & $22.19 \pm 2.85$ & $22.36 \pm 2.89$ & $21.07 \pm 3.18$ & 0.001 \\
\hline \multicolumn{5}{|l|}{ Severity of AP } \\
\hline MAP & 150 (31.7\%) & 119 (30.3\%) & 31 (38.8\%) & 0.312 \\
\hline MSAP & $190(40.2 \%)$ & 160 (40.7\%) & $30(37.5 \%)$ & \\
\hline SAP & $133(28.1 \%)$ & $114(29.0 \%)$ & $19(23.8 \%)$ & \\
\hline \multicolumn{5}{|l|}{ Aetiology of AP } \\
\hline Biliary & $384(81.2 \%)$ & $313(79.6 \%)$ & 71 (88.6\%) & 0.203 \\
\hline HTG & $17(3.6 \%)$ & $16(4.1 \%)$ & I (I.3\%) & \\
\hline Alcohol & $20(4.2 \%)$ & $19(4.8 \%)$ & $\mathrm{I}(\mathrm{I} .3 \%)$ & \\
\hline Other & $52(11.0 \%)$ & 45 (II.5\%) & $7(8.8 \%)$ & \\
\hline \multicolumn{5}{|l|}{ Hypertension } \\
\hline Yes & $169(35.7 \%)$ & $134(34.1 \%)$ & $35(43.8 \%)$ & 0.101 \\
\hline No & $304(64.3 \%)$ & 259 (65.9\%) & $45(56.3 \%)$ & \\
\hline \multicolumn{5}{|l|}{ Diabetes } \\
\hline Yes & 37 (7.8\%) & 31 (92.1\%) & $6(7.5 \%)$ & 0.906 \\
\hline No & $436(92.2 \%)$ & $362(92.1 \%)$ & $74(92.5 \%)$ & \\
\hline \multicolumn{5}{|l|}{ Chronic renal failure } \\
\hline Yes & II (2.3\%) & $10(2.5 \%)$ & 79 (98.8\%) & 0.484 \\
\hline No & $462(97.7 \%)$ & $383(97.5 \%)$ & I (I.3\%) & \\
\hline \multicolumn{5}{|l|}{ Smoking } \\
\hline Yes & 101 (2I.4\%) & 90 (22.9\%) & II (13.8\%) & 0.069 \\
\hline No & $372(78.6 \%)$ & $303(77.1 \%)$ & $69(86.3 \%)$ & \\
\hline \multicolumn{5}{|l|}{ Drinking } \\
\hline Yes & $84(17.8 \%)$ & 73 (18.6\%) & II (I3.8\%) & 0.303 \\
\hline No & 389 (82.2\%) & $320(81.4 \%)$ & 69 (86.3\%) & \\
\hline \multicolumn{5}{|l|}{ Complications } \\
\hline \multicolumn{5}{|l|}{ Organ failure } \\
\hline Acute respiratory failure & $120(25.4 \%)$ & $102(26.0 \%)$ & 18 (22.5\%) & 0.517 \\
\hline Acute renal failure & $57(12.1 \%)$ & 44 (II.2\%) & $13(16.3 \%)$ & 0.206 \\
\hline Shock & $23(4.9 \%)$ & $22(5.6 \%)$ & I (I.3\%) & 0.099 \\
\hline Multi-organ failure & 45 (9.5\%) & 35 (8.9\%) & $10(12.5 \%)$ & 0.318 \\
\hline \multicolumn{5}{|l|}{$\begin{array}{l}\text { Pancreatic } \\
\text { complications }\end{array}$} \\
\hline ANC & $31(6.6 \%)$ & $26(6.6 \%)$ & $5(6.3 \%)$ & 0.073 \\
\hline ANC infection & $28(5.9 \%)$ & $26(6.6 \%)$ & $2(2.5 \%)$ & \\
\hline APFC & $123(26.0 \%)$ & $108(27.5 \%)$ & 15 (I8.8\%) & \\
\hline APFC infection & 14 (3.0\%) & $13(3.3 \%)$ & I (I.3\%) & \\
\hline WON & $6(1.3 \%)$ & $4(1.3 \%)$ & $2(2.5 \%)$ & \\
\hline WON infection & $4(0.8 \%)$ & $2(0.5 \%)$ & 2 (2.5\%) & \\
\hline PPC & $2(0.4 \%)$ & I (0.3\%) & I (I.3\%) & \\
\hline
\end{tabular}

(Continued) 
Table I (Continued).

\begin{tabular}{|c|c|c|c|c|}
\hline & All Patients $(n=473)$ & Older Group (60-80 Years) $(n=393)$ & Oldest Group ( $\geq 80$ years) $(n=80)$ & $\mathbf{P}$ \\
\hline \multicolumn{5}{|l|}{ Operative treatment } \\
\hline ERCP & $103(21.8 \%)$ & 81 (20.6\%) & $22(27.5 \%)$ & 0.174 \\
\hline PCD & II (24.7\%) & $93(23.7 \%)$ & $24(30.0 \%)$ & 0.231 \\
\hline Cholecystectomy & $19(4.0 \%)$ & $19(4.8 \%)$ & $0(0.0 \%)$ & 0.097 \\
\hline Necrosectomy & $32(6.8 \%)$ & $28(7.1 \%)$ & $4(5.0 \%)$ & 0.097 \\
\hline \multicolumn{5}{|l|}{ Symptoms } \\
\hline Abdominal pain & $278(58.8 \%)$ & $24 \mid(61.3 \%)$ & $37(46.3 \%)$ & 0.013 \\
\hline Vomiting & $34(7.2 \%)$ & $26(6.6 \%)$ & $8(10 \%)$ & 0.285 \\
\hline Jaundice & $49(10.4 \%)$ & $35(8.9 \%)$ & $14(17.5 \%)$ & 0.021 \\
\hline Dyspnoea & $66(14.0 \%)$ & 45 (1 I.5\%) & $21(26.3 \%)$ & 0.001 \\
\hline \multicolumn{5}{|l|}{ ICU admission } \\
\hline Yes & $159(33.6 \%)$ & $129(32.8 \%)$ & 30 (37.5\%) & 0.42 \\
\hline No & $314(66.4 \%)$ & 264 (67.2\%) & 50 (62.5\%) & \\
\hline LOS, days & $12.1 \pm 10.4$ & $11.5 \pm 10.2$ & $15.3 \pm 11.0$ & 0.003 \\
\hline \multicolumn{5}{|l|}{ Death } \\
\hline Yes & $48(10.1 \%)$ & 35 (8.9\%) & $13(16.3 \%)$ & 0.047 \\
\hline No & 425 (89.9\%) & 358 (91.1\%) & 67 (83.8\%) & \\
\hline
\end{tabular}

Notes: Continuous variables are presented as the mean (standard deviation). Categorical variables are presented as number (percentage).

Abbreviations: AP, acute pancreatitis; ARF, acute respiratory failure; LOS, length of stay; ERCP, endoscopic retrograde cholangiopancreatography; ICU, intensive care unit; BMI, body mass index; AKI, acute kidney injury; MAP, mild acute pancreatitis; MSAP, moderately severe acute pancreatitis; SAP, severe acute pancreatitis; HTG, hypertriglyceridemia; ERCP, endoscopic retrograde cholangiopancreatography; PPC, pancreatic pseudocyst; WON, walled-off necrosis; APFC, acute peripancreatic fluid collection; ANC, acute necrosis collection; PCD, percutaneous drainage; HTG, hypertriglyceridemia.

Table 2 Comparison of the Laboratory Tests and Scores of AP Patients in the Older Group and Oldest Group

\begin{tabular}{|c|c|c|c|c|}
\hline & All AP Patients $(n=473)$ & Older Group (60-80 Years) $(n=393)$ & Oldest Group ( $\geq 80$ Years) $(n=80)$ & $\mathbf{P}$ \\
\hline WBC $\left(10^{9} / L\right)$ & $11.9 \pm 5.7$ & $11.9 \pm 5.7$ & II. $.4 \pm 5.8$ & 0.407 \\
\hline LDH (U/L) & $4 \mid 3.1 \pm 307.2$ & $421.2 \pm 325.0$ & $373.5 \pm 194.4$ & 0.206 \\
\hline AST (U/L) & $90.0 \pm 123.4$ & $90.9 \pm 123.8$ & $85.8 \pm 122.2$ & 0.736 \\
\hline ALT (U/L) & $97.9 \pm 125.3$ & $102.0 \pm 132.2$ & $75.9 \pm 89.4$ & 0.085 \\
\hline ALB (g/L) & $35.3 \pm 4.9$ & $34.9 \pm 4.6$ & $35.4 \pm 5.0$ & 0.459 \\
\hline AMY (U/L) & $496.4 \pm 593.6$ & $501.0 \pm 599.1$ & $473.5 \pm 568.7$ & 0.706 \\
\hline GLU (mmol/L) & $8.4 \pm 4.4$ & $8.3 \pm 4.3$ & $8.5 \pm 5.2$ & 0.771 \\
\hline TG (mmol/L) & $1.4 \pm 2.9$ & $1.5 \pm 3.1$ & $1.1 \pm 0.6$ & 0.219 \\
\hline TC (mmol/L) & $3.9 \pm 1.6$ & $3.9 \pm 1.7$ & $3.8 \pm 1.0$ & 0.409 \\
\hline TBIL ( $\mu \mathrm{mol} / \mathrm{L})$ & $34.4 \pm 34.7$ & $34.3 \pm 34.8$ & $34.8 \pm 34.5$ & 0.902 \\
\hline DBIL ( $\mu \mathrm{mol} / \mathrm{L})$ & $20.4 \pm 30.9$ & $20.2 \pm 31.4$ & $20.9 \pm 28.8$ & 0.857 \\
\hline BUN (mmol/L) & $7.2 \pm 5.9$ & $7.2 \pm 6.1$ & $7.2 \pm 4.7$ & 0.958 \\
\hline $\mathrm{Cr}(\mu \mathrm{mol} / \mathrm{L})$ & $88.6 \pm 76.7$ & $88.1 \pm 79.6$ & $91.5 \pm 60.3$ & 0.719 \\
\hline CRP (mg/L) & $122.6 \pm 94.6$ & $126.2 \pm 96.5$ & $104.6 \pm 82.8$ & 0.063 \\
\hline APACHE II & $12.1 \pm 3.7$ & $11.9 \pm 3.7$ & $13.1 \pm 3.5$ & 0.010 \\
\hline APACHE II-age & $7.4 \pm 3.9$ & $7.4 \pm 3.6$ & $7.1 \pm 3.5$ & 0.443 \\
\hline BISAP & $1.9 \pm 1.2$ & $1.9 \pm 1.2$ & $2.1 \pm 1.2$ & 0.317 \\
\hline BISAP-age & $1.0 \pm 1.1$ & $0.9 \pm 1.1$ & $1.1 \pm 1.2$ & 0.567 \\
\hline Marshall & $1.4 \pm 1.3$ & $1.4 \pm 1.3$ & $1.4 \pm 1.2$ & 0.379 \\
\hline Glasgow & $0.1 I \pm 0.3$ & $0.5 \pm 0.3$ & $0.1 \pm 0.4$ & 0.156 \\
\hline
\end{tabular}

Note: Continuous variables are presented as the mean (standard deviation).

Abbreviations: AP, acute pancreatitis; WBC, white blood cells; LDH, lactic dehydrogenase; ALT, alanine aminotransferase; AST, aspartate transaminase; TBIL, total bilirubin; DBIL, direct bilirubin; CRP, C-reactive protein; BUN, urea nitrogen; GLU, glucose; TC, total cholesterol; TG, triglycerides; ALB, albumin; AMY, amylase; Cr, creatinine; APACHE II, Acute Physiology and Chronic Health Evaluation II; BISAP, bedside index for severity in acute pancreatitis; HTG, hypertriglyceridemia. 
On CECT, acute necrosis collection (ANC) was detected in $26(6.6 \%)$ patients in the older group compared to $5(6.3 \%)$ patients in the oldest group. The older group was more likely than the oldest group to have acute peripancreatic fluid collection (APFC) and pancreatic complications (108 and 15 in the older and oldest cohorts, respectively). Walled-off necrosis (WON) was found in 4 $(1.3 \%)$ and $2(2.5 \%)$ patients in the older group and oldest group, respectively. Infection due to ANC, APFC and WON seldom occurred, and there were significant differences between the groups.

Operative treatment is an approach to AP. Endoscopic retrograde cholangiopancreatography (ERCP) was performed equally in both ageing groups $(20.6 \%$ older group and $27.5 \%$ oldest patients, $\mathrm{P}=0.174$ ). Percutaneous drainage (PCD) was performed slightly more often in the oldest patients than in the older patients ( $23.7 \%$ older patients vs $30.0 \%$ oldest patients), but there were no differences between the two groups $(\mathrm{P}=0.231)$. Necrosectomy was performed in $28(7.1 \%)$ AP patients in the older group and $4(5.0 \%)$ in the oldest group. Interestingly, cholecystectomy was only performed in the older group (4.8\%).

A longer length of hospital stay was also found for the oldest group than for the older group $(11.51 \pm 10.19$ vs $11.51 \pm 10.19, \mathrm{P}=0.003)$. Conversely, there was no difference in ICU admission between the two groups of patients ( $32.8 \%$ older group vs $37.5 \%$ oldest group). The death rate was higher in the oldest group (16.3\%) than in the older group $(8.9 \%)$, with a significant difference $(\mathrm{P}=0.047)$. Moreover, multiple organ failure occurred more frequently in the oldest group than in the older group $(12.5 \%$ vs $8.9 \%)$. Regarding acute respiratory failure, there were 18 cases $(22.5 \%)$ in the older group and $102(26.0 \%)$ in the oldest group. Acute renal failure occurred in 13 (16.3\%) patients in the oldest group and $44(11.2 \%)$ in the older group. Surprisingly, 22 (5.6\%) of the older patients experienced shock, but only $1(1.3 \%)$ patient in the older group did. There was no difference in organ failure between the two groups in this study.

\section{Univariate and Multivariate Regression Analyses of Risk Factors for Clinical Outcomes \\ Death Rate (Details are Listed in Table 3)}

First, univariate analysis was performed to evaluate the risk factors for death. The AP patients aged $\geq 80$ years experienced higher rates of death $(\mathrm{OR}, 1.06,95 \% \mathrm{CI}$, $1.02-1.10, \mathrm{P}=0.003)$ and $\mathrm{SAP}$, a more severe AP presentation (OR, 17.50, 95\% CI, 5.24-58.48, $\mathrm{P}=0.001$ ), and higher rates of ERCP (OR, 0.22, 95\% CI, 0.56-2.84, $\mathrm{P}=0.012)$, organ failure $(\mathrm{P}=0.001)$ and local complications of ANC and ANC infection $(\mathrm{P}=0.001)$, with significantly different clinical presentations.

In the multivariate analysis, age over 80 years (OR, $3.30,95 \% \mathrm{CI}, 1.32-8.27, \mathrm{P}=0.011)$, acute respiratory failure (OR, 4.23, 95\% CI, 1.32-13.50, $\mathrm{P}=0.015$ ), shock (OR, 4.95, 95\% CI, 1.58-15.57, $\mathrm{P}=0.006$ ), and multi-organ failure (OR, 4.61, 95\% CI, 1.39-15.34, $\mathrm{P}=$ 0.013 ) were all verified as independent risk factors for a higher mortality rate.

\section{ICU Admission (Details are Listed in Table 4)}

BMI (OR, 1.13, 95\% CI, 1.06-1.21, P =0.001), SAP, a more severe AP presentation (OR, 38.04, 95\% CI, 19.2575.17, $\mathrm{P}=0.001$ ), hypertriglyceridemia aetiology (OR, $1.55,95 \% \mathrm{CI}, 0.07-4.28, \mathrm{P}=0.026$ ), absence of typical AP symptomatology of abdominal pain (OR, 2.22, 95\% CI, 1.48-3.34, $\mathrm{P}=0.001$ ), necrosectomy (OR, 2.39, 95\% $\mathrm{CI}, \quad 1.16-4.92, \quad \mathrm{P}=0.018$ ), acute respiratory failure $(\mathrm{P}=0.001)$ and local complications $(\mathrm{P}=0.001)$ were significantly associated with a higher ICU admission rate in the univariate analysis. However, in the multivariate analysis, ICU admission was associated with a more severe AP presentation (OR, 11.72, 95\% CI, 4.78-28.76, P =0.001), the absence of abdominal pain (OR, 1.91, 95\% CI, 1.05$3.45, \mathrm{P}=0.033$ ), acute respiratory failure ( $\mathrm{OR}, 4.18,95 \%$ $\mathrm{CI}, 1.85-9.44, \mathrm{P}=0.001$ ), and multiorgan failure (OR, 5.10, 95\% CI, 1.27-20.50, $\mathrm{P}=0.022$ ).

\section{Length of Stay (LOS) (Details are Listed in Table 5)}

Univariate analysis revealed many risk factors related to LOS, such as age (OR, 1.11, 95\% CI, 0.02-0.27, P $=0.023)$, female sex (OR, 0.09, 95\% CI, 0.03-3.80, P $=0.046)$, severity of AP $(\mathrm{OR}, 0.36,95 \% \mathrm{CI}, 3.76-6.03, \mathrm{P}$ $=0.001)$, hypertension $(\mathrm{OR}, 0.10,95 \% \mathrm{CI}, 0.11-4.03, \mathrm{P}$ $=0.038)$, diarrhoea $(\mathrm{OR}, 0.15,95 \% \mathrm{CI}, 1.56-6.20, \mathrm{P}$ $=0.001)$, jaundice $(\mathrm{OR}, 0.18,95 \% \mathrm{CI}, 1.62-7.75, \mathrm{P}$ $=0.003)$, dyspnoea (OR, 1.37, 95\% CI, 2.16-7.53, P $=0.001), \mathrm{PCD}(\mathrm{OR}, 0.22,95 \% \mathrm{CI}, 3.06-7.33, \mathrm{P}=0.001)$, necrosectomy (OR, $0.31,95 \% \mathrm{CI}, 9.17-16.32, \mathrm{P}=0.001$ ), organ failure $(\mathrm{P}=0.001)$ and local complications ( $\mathrm{OR}, 0.28$ $95 \% \mathrm{CI}, 0.43-9.17, \mathrm{P}=0.001)$. In the multivariate analysis, age (OR, 0.15, 95\% CI, 2.03-6.27, $\mathrm{P}=0.001$ ), female sex (OR, $0.09,95 \% \mathrm{CI}, 0.34-3.54, \mathrm{P}=0.018$ ), severity of AP 
Table 3 Univariate and Multivariate Analyses of Risk Factors for Mortality in Ageing Patients with Acute Pancreatitis

\begin{tabular}{|c|c|c|c|c|}
\hline \multirow[t]{2}{*}{ Variables } & \multicolumn{2}{|c|}{ Univariate Analysis } & \multicolumn{2}{|c|}{ Multivariate Analysis } \\
\hline & OR (95\% Cl) & $P$ & OR (95\% CI) & $P$ \\
\hline Age (years) & $1.06(1.02-1.10)$ & 0.003 & $3.30(1.32-8.27)$ & 0.011 \\
\hline Sex (male/female) & $1.06(1.02-1.10)$ & 0.546 & & \\
\hline BMI & $1.03(0.94-1.14)$ & 0.512 & & \\
\hline \multicolumn{5}{|l|}{ Severity of AP } \\
\hline MAP & $\operatorname{Ref}(1.00)$ & & $\operatorname{Ref}(1.00)$ & \\
\hline MSAP & $2.72(0.74-10.08)$ & 0.134 & $1.58(0.35-7.18)$ & 0.546 \\
\hline SAP & 17.50 (5.24-58.48) & 0.001 & $2.37(0.45-12.43)$ & 0.307 \\
\hline \multicolumn{5}{|l|}{ Aetiology of AP } \\
\hline Biliary & $\operatorname{Ref}(1.00)$ & & & \\
\hline HTG & $0.55(0.07-4.28)$ & 0.570 & & \\
\hline Alcohol & I.56 (0.44-5.57) & 0.492 & & \\
\hline Other & $0.94(0.35-2.5 I)$ & 0.903 & & \\
\hline Hypertension & $1.32(0.72-2.43)$ & 0.366 & & \\
\hline Diabetes & $0.48(0.11-2.08)$ & 0.330 & & \\
\hline Chronic renal failure & $0.88(0.11-7.05)$ & 0.907 & & \\
\hline History of smoking & I.II (0.54-2.26) & 0.780 & & \\
\hline History of drinking & $1.43(0.70-2.94)$ & 0.326 & & \\
\hline \multicolumn{5}{|l|}{ Symptoms } \\
\hline Abdominal pain & I.3I (0.7I-2.45) & 0.389 & & \\
\hline Vomiting & I.35 (0.68-2.7I) & 0.394 & & \\
\hline Jaundice & $1.27(0.52-3.16)$ & 0.608 & & \\
\hline Dyspnoea & $1.27(0.56-2.84)$ & 0.568 & & \\
\hline \multicolumn{5}{|l|}{ Operative treatment } \\
\hline ERCP & $0.22(0.56-2.84)$ & 0.012 & $0.28(0.07-1.12)$ & 0.072 \\
\hline PCD & I.I5 (0.58-2.25) & 0.691 & & \\
\hline Cholecystectomy & $0.00(0.00-)$ & 0.998 & & \\
\hline Necrosectomy & $1.29(0.43-3.85)$ & 0.649 & & \\
\hline \multicolumn{5}{|l|}{ Organ failure } \\
\hline Acute respiratory failure & 13.86 (6.78-28.32) & 0.001 & $4.23(1.32-13.50)$ & 0.015 \\
\hline Acute renal failure & $15.02(7.65-29.5 \mathrm{I})$ & 0.001 & $0.94(0.27-3.36)$ & 0.927 \\
\hline Shock & $23.69(9.36-59.95)$ & 0.001 & $4.95(1.58-15.57)$ & 0.006 \\
\hline Multi-organ failure & $22.01(10.69-45.34)$ & 0.001 & $4.61(1.39-15.34)$ & 0.013 \\
\hline \multicolumn{5}{|l|}{ Local complication } \\
\hline ANC & $5.80(2.22-15.12)$ & 0.001 & $0.87(0.23-3.28)$ & 0.837 \\
\hline ANC infection & $9.26(3.65-23.52)$ & 0.001 & $0.88(0.3 \mathrm{I}-2.49)$ & 0.812 \\
\hline APFC & $1.80(0.82-3.98)$ & 0.145 & $0.46(0.04-5.33)$ & 0.532 \\
\hline APFC infection & $1.28(0.16-10.47)$ & 0.817 & $1.35(0.36-5.04)$ & 0.655 \\
\hline WON & $3.33(0.37-30.37)$ & 0.285 & $1.26(0.09-17.77)$ & 0.865 \\
\hline WON infection & $5.56(0.55-56.67)$ & 0.148 & $6.70(0.50-89.74)$ & 0.151 \\
\hline PPC & $0.00(0.00-)$ & 0.99 & $0.00(0.00-)$ & 0.999 \\
\hline
\end{tabular}

Abbreviations: AP, acute pancreatitis; ARF, acute respiratory failure; LOS, length of stay; ERCP, endoscopic retrograde cholangiopancreatography; ICU, intensive care unit; BMI, body mass index; AKI, acute kidney injury; MAP, mild acute pancreatitis; MSAP, moderately severe acute pancreatitis; SAP, severe acute pancreatitis; OR, odds ratio; ERCP, endoscopic retrograde cholangiopancreatography; PPC, pancreatic pseudocyst; WON, walled-off necrosis; APFC, acute peripancreatic fluid collection; ANC, acute necrosis collection; PCD, percutaneous drainage; HTG, hypertriglyceridemia. 
Table 4 Univariate and Multivariate Analyses of Risk Factors for ICU Admission in Ageing Patients with Acute Pancreatitis

\begin{tabular}{|c|c|c|c|c|}
\hline \multirow[t]{2}{*}{ Variables } & \multicolumn{2}{|c|}{ Univariate Analysis } & \multicolumn{2}{|c|}{ Multivariate Analysis } \\
\hline & OR (95\% Cl) & $\mathbf{P}$ & OR (95\% Cl) & $P$ \\
\hline Age (years) & $1.23(0.75-2.20)$ & 0.402 & & \\
\hline Sex (male/female) & $0.93(0.63-1.36)$ & 0.688 & & \\
\hline BMI & $1.13(1.06-1.21)$ & 0.001 & $0.99(0.90-1.09)$ & 0.812 \\
\hline \multicolumn{5}{|l|}{ Severity of AP } \\
\hline MAP & Ref $(1.00)$ & & $\operatorname{Ref}(1.00)$ & \\
\hline MSAP & $1.83(0.97-3.45)$ & 0.064 & & \\
\hline SAP & 38.04 (19.25-75.17) & 0.001 & II.72 (4.78-28.76) & 0.001 \\
\hline \multicolumn{5}{|l|}{ Aetiology of AP } \\
\hline Biliary & $\operatorname{Ref}(1.00)$ & & $\operatorname{Ref}(1.00)$ & \\
\hline HTG & $1.55(0.07-4.28)$ & 0.026 & $2.11(0.52-8.63)$ & 0.298 \\
\hline Alcohol & $1.56(0.44-5.57)$ & 0.013 & $2.56(0.7 I-9.25)$ & 0.152 \\
\hline Other & $0.94(0.35-2.5 \mathrm{I})$ & 0.670 & & \\
\hline Hypertension & $1.64(\mathrm{I} .1 \mathrm{I}-2.43)$ & 0.014 & $1.45(0.83-2.53)$ & 0.198 \\
\hline Diabetes & $1.22(0.6 \mathrm{I}-2.44)$ & 0.572 & & \\
\hline Chronic renal failure & $0.74(0.19-2.8 I)$ & 0.654 & & \\
\hline History of smoking & $0.90(0.60-1.43)$ & 0.643 & & \\
\hline History of drinking & $1.27(0.80-2.07)$ & 0.339 & & \\
\hline \multicolumn{5}{|l|}{ Symptoms } \\
\hline Abdominal pain & $2.22(1.48-3.34)$ & 0.001 & $1.91(1.05-3.45)$ & 0.033 \\
\hline Vomiting & I.84 (0.9I-3.7I) & 0.089 & & \\
\hline Diarrhoea & $1.24(0.78-1.97)$ & 0.357 & & \\
\hline Jaundice & $1.55(0.85-2.84)$ & 0.150 & & \\
\hline Dyspnoea & $1.44(0.85-2.46)$ & 0.178 & & \\
\hline \multicolumn{5}{|l|}{ Operative treatment } \\
\hline ERCP & $0.60(0.0 .37-0.99)$ & 0.043 & $1.34(0.69-2.59)$ & 0.391 \\
\hline PCD & $1.14(0.74-1.77)$ & 0.547 & & \\
\hline Cholecystectomy & $0.5 \mid(0.17-1.58)$ & 0.245 & & \\
\hline Necrosectomy & $2.39(1.16-4.92)$ & 0.018 & $0.84(0.25-2.86)$ & 0.780 \\
\hline \multicolumn{5}{|l|}{ Organ failure } \\
\hline Acute respiratory failure & $18.4 \mid$ (10.9|-31.09) & 0.001 & $4.18(1.85-9.44)$ & 0.001 \\
\hline Acute renal failure & 14.67 (6.93-30.85) & 0.001 & $0.87(0.24-2.89)$ & 0.765 \\
\hline Shock & $372(0.000-)$ & 0.998 & & \\
\hline Multi-organ failure & $26.93(9.44-76.82)$ & 0.001 & $5.10(1.27-20.50)$ & 0.022 \\
\hline \multicolumn{5}{|l|}{ Local complications } \\
\hline ANC & $5.29(2.44-11.45)$ & 0.001 & $0.59(0.18-2.02)$ & 0.403 \\
\hline ANC infection & $2.62(1.64-4.17)$ & 0.001 & $3.10(0.68-14.11)$ & 0.144 \\
\hline APFC & $5.09(1.7-15.28)$ & 0.004 & $0.89(0.43-1.84)$ & 0.760 \\
\hline APFC infection & 22.91 (7.63-68.78) & 0.001 & $3.15(0.73-13.54)$ & 0.124 \\
\hline WON & $3.82(0.75-19.44)$ & 0.107 & & \\
\hline WON infection & $1.27(0.13-12.48)$ & 0.836 & & \\
\hline PPC & $0.00(0.00)$ & 0.999 & & \\
\hline
\end{tabular}

Abbreviations: AP, acute pancreatitis; ARF, acute respiratory failure; LOS, length of stay; ERCP, endoscopic retrograde cholangiopancreatography; ICU, intensive care unit; BMI, body mass index; AKI, acute kidney injury; MAP, mild acute pancreatitis; MSAP, moderately severe acute pancreatitis; SAP, severe acute pancreatitis; OR, odds ratio; ERCP, endoscopic retrograde cholangiopancreatography; PPC, pancreatic pseudocyst; WON, walled-off necrosis; APFC, acute peripancreatic fluid collection; ANC, acute necrosis collection; PCD, percutaneous drainage; HTG, hypertriglyceridemia. 
Table 5 Univariate and Multivariate Analyses of the Risk Factors for Length of Hospital Stay in Ageing Patients with Acute Pancreatitis

\begin{tabular}{|c|c|c|c|c|}
\hline \multirow[t]{2}{*}{ Variables } & \multicolumn{2}{|c|}{ Univariate Analysis } & \multicolumn{2}{|l|}{ Multivariate Analysis } \\
\hline & $\beta(95 \% \mathrm{Cl})$ & $P$ value & $\beta(95 \% \mathrm{Cl})$ & $P$ value \\
\hline Age (years) & I.I I $(0.02-0.27)$ & 0.023 & $0.15(2.03-6.27)$ & 0.001 \\
\hline Sex (male/female) & $0.09(0.03-3.80)$ & 0.046 & $0.09(0.34-3.54)$ & 0.018 \\
\hline BMI & $0.06(0.00-0.54)$ & 0.173 & & \\
\hline Severity of AP & $0.36(3.76-6.03)$ & 0.001 & $0.22(1.57-4.32)$ & 0.001 \\
\hline Aetiology of AP & $0.01(-0.86-1.04)$ & 0.188 & & \\
\hline Hypertension & $0.10(0.11-4.03)$ & 0.038 & $0.06(-0.3|-2.9|)$ & 0.113 \\
\hline Diabetes & $0.03(-2.50-4.52)$ & 0.571 & & \\
\hline Chronic renal failure & $-0.01(-6.88-5.64)$ & 0.847 & & \\
\hline History of smoking & $-0.03(-3.17-1.43)$ & 0.459 & & \\
\hline History of drinking & $0.02(-1.88-3.06)$ & 0.640 & & \\
\hline \multicolumn{5}{|l|}{ Symptoms } \\
\hline Abdominal pain & $0.03(-|.32-2.5|)$ & 0.544 & & \\
\hline Vomiting & $0.03(-2.38-4.92)$ & 0.496 & & \\
\hline Diarrhoea & $0.15(1.56-6.20)$ & 0.001 & $0.07(-0.11-3.89)$ & 0.064 \\
\hline Jaundice & $0.18(1.62-7.75)$ & 0.003 & $0.08(0.15-5.32)$ & 0.038 \\
\hline Dyspnoea & $1.37(2.16-7.53)$ & 0.001 & $0.07(-0.14-4.43)$ & 0.065 \\
\hline \multicolumn{5}{|l|}{ Operative treatment } \\
\hline ERCP & $0.001(-2.25-2.32)$ & 0.977 & & \\
\hline Percutaneous drainage & $0.22(3.06-7.33)$ & 0.001 & $0.21(5.34-11.67)$ & 0.001 \\
\hline Cholecystectomy & $-0.04(-6.7 \mid-2.89)$ & 0.435 & & \\
\hline Necrosectomy & $0.31(9.17-16.32)$ & 0.001 & 0.21 (5.34-I I.68) & 0.001 \\
\hline \multicolumn{5}{|l|}{ Organ failure } \\
\hline Acute respiratory failure & $0.35(6.28-10.34)$ & 0.001 & $0.14(0.8 I-6.03)$ & 0.010 \\
\hline Acute renal failure & $0.22(4.33-9.98)$ & 0.001 & $0.02(-3.14-4.46)$ & 0.733 \\
\hline Shock & $0.31(10.38-18.74)$ & 0.001 & $0.23(6.93-15.22)$ & 0.001 \\
\hline Multi-organ failure & $0.14(1.66-8.03)$ & 0.003 & $-0.18(-10.43-2.45)$ & 0.200 \\
\hline Local complications & $0.28(1.43-2.70)$ & 0.001 & $0.07(-0.09-1.16)$ & 0.093 \\
\hline
\end{tabular}

Abbreviations: AP, acute pancreatitis; ARF, acute respiratory failure; LOS, length of stay; ERCP, endoscopic retrograde cholangiopancreatography; ICU, intensive care unit; BMI, body mass index; AKI, acute kidney injury; MAP, mild acute pancreatitis; MSAP, moderately severe acute pancreatitis; SAP, severe acute pancreatitis; OR, odds ratio; ERCP, endoscopic retrograde cholangiopancreatography; PPC, pancreatic pseudocyst; WON, walled-off necrosis; APFC, acute peripancreatic fluid collection; ANC, acute necrosis collection; PCD, percutaneous drainage; HTG, hypertriglyceridemia.

(OR, 0.22, 95\% CI, 1.57-4.32, $\mathrm{P}=0.001)$, diarrhoea $(\mathrm{OR}$, $0.07,95 \% \mathrm{CI},-0.11-3.89, \mathrm{P}=0.064)$, jaundice $(\mathrm{OR}, 0.08$, 95\% CI, 0.15-5.32, P =0.038), PCD (OR, 0.21, 95\% CI, $5.34-11.68, \mathrm{P}=0.001)$, necrosectomy $(\mathrm{OR}, 0.21,95 \% \mathrm{CI}$, $5.34-11.68, \mathrm{P}=0.001)$, acute respiratory failure $(\mathrm{OR}, 0.14$, 95\% CI, 0.81-6.03, P =0.010), and shock (OR, 0.230, 95\% $\mathrm{CI}, 6.93-15.22, \mathrm{P}=0.001)$ were independent risk factors for a longer LOS.

\section{Discussion}

With a great increase in the ageing population worldwide, there has been an increase in the number of age-associated acute and chronic diseases. AP is an acute disease, and the incidence rate increases proportionally with age. ${ }^{24}$ Data from an emergency department (ED) showed that ageing patients aged over 65 years old diagnosed with AP comprised one-third of all patients. ${ }^{8}$ Another registry study reported a six-fold increase in the incidence of AP patients aged over 80 years over a decade. ${ }^{25}$

Although we have paid attention to the importance of the clinical outcomes of the frail portion of the AP population, only a small number of studies with limited data have been performed, and they have yielded conflicting results. A study from Europe reported an increased morbidity rate for AP patients aged $\geq 65$ years, especially those $\geq 80$ years. ${ }^{4}$ However, Kim JE.el found no significant difference in the clinical course of AP between older and younger patients. $^{15}$ The difference between those studies is 
probably due to the age brackets established by the different authors. Most previous authors have concluded that ageing patients generally develop more severe AP and that age may be regarded as a risk factor for clinical outcomes. To resolve this controversial issue, we compared the clinical outcomes of ageing AP patients divided into an older group (60-79 years old) and an oldest group ( $\geq 80$ years old) who were admitted to our pancreas centre at the First Affiliated Hospital of Nanchang University of China. Given the increased life expectancy of ageing patients in China, the age cut-off chosen in our study encompass a large proportion of ageing patients in the near future.

In this study, we aimed to analyse age-associated clinical outcomes and assess risk factors for ageing patients with AP. Interestingly, our study determined that age over 80 years significantly influenced the mortality rate and length of hospitalization, although the ICU admission rate was similar to that of the older group.

With regard to the clinical presentation of ageing AP patients, more severe manifestations were found in the oldest group than in the older group in our study. This has also been reported in previous research showing that increasing age is closely related to a more severe clinical course of AP. ${ }^{15-18,26}$ The underlying mechanism of the above conditions is still under debate. One viewpoint proposed in the literature is that increasing age is associated with the loss of protective proteins, leading to an adverse clinical course in the oldest patients. ${ }^{27}$ Pre-existing comorbidities such as diabetes and chronic renal disease also influence the physiological functions of ageing AP patients, causing a more severe inflammatory response, as reflected by the primary clinical manifestation of the disease. $^{13}$

According to clinical experience, the clinical assessment of ageing patients is usually more difficult than that of younger patients. ${ }^{28}$ Our research verified the absence of typical symptoms in $28 \%$ of our ageing patients. This finding serves as a reminder of the need for increased attention to ageing AP patients, especially those with more severe disease. In our study, we found that abnormal symptomatology was present in ageing AP patients. In this regard, abdominal pain occurred less frequently in the oldest group than in the older group, which is in line with previous studies that reported a decline in abdominal pain in ageing patients over 80 years old. This may be linked to a greater capacity to endure pain and to reduced feedback. In our study, we found for the first time that abdominal pain was significantly related to the ICU admission rate in ageing patients. In contrast, clinical symptoms were not associated with the death rate, which was not in line with a previous study that showed that the death rate is closely related to abdominal pain. ${ }^{13} \mathrm{We}$ speculate that our findings may be attributable to the full treatment experience provided for more severe ageing AP patients at our hospital, which is one of the largest pancreas centres in China, leading to a higher ICU admission rate and lower death rate.

In addition, an interesting phenomenon of symptoms was found in our study: dyspnoea, jaundice and diarrhoea occurred more frequently in the oldest group than in the older group $(\mathrm{P}<0.05)$. The relationship between AP and dyspnoea symptoms in ageing patients has already been reported. ${ }^{21}$ Our findings for the other two symptoms, jaundice and diarrhoea, were not in line with previous research reporting that the rates of those symptoms are similar in older and oldest groups. We suspect that age itself is the explanation for the diversity of symptoms in ageing people with AP. In addition, our study surprisingly found that jaundice symptoms are related to LOS, which has not been reported by other studies. These conditions remind us that we should pay more attention to symptoms of diarrhoea, jaundice, and dyspnoea in the absence of abdominal pain of AP in ageing patients.

A previous study reported that a lower BMI ranging from 18.5 to 19.9 was associated with a higher risk of death relative to the population with a BMI over 21.5. ${ }^{29}$ Another study found that BMI less than 20.0 was associated with an increased risk of death, whereas a BMI $\geq$ 23.0 was not associated with a higher death rate. ${ }^{30}$ In our study, a lower BMI (21.07 \pm 3.18$)$ was found in the oldest group than in the older group (22.36 \pm 2.89$)$, and there were significant differences between the two groups. Although BMI was not an independent risk factor for mortality in our study, the combination of age and BMI was associated with the risk of death. One potential explanation for this may relate to energy and nutritional reserves. Malnutrition has been verified as a predictor of death, and people with less energy and nutritional reserves are less resistant to infection. ${ }^{31}$ Hence, ageing patients should consume appropriate nutrition to increase their BMI and improve their resistance to disease.

Overexpression of interleukin (IL)-6, IL-8 and tumour necrosis factor (TNF)- $\alpha$ was verified after the onset of AP, which is a known proinflammatory status. Proinflammation facilitates systemic symptoms, especially respiratory and renal injury, as the first manifestation of 
AP. In particular, IL-6, TNF- $\alpha$ and alveolar macrophages accumulate after neutrophils are activated in the lungs, after which a second wave of inflammation develops, causing the onset of respiratory symptoms as the first sign of disease. ${ }^{32}$ Depending the condition, intensive care may be necessary to guarantee sufficient cardiopulmonary monitoring and suitable electrolyte and fluid replacement for ageing patients after AP onset. ${ }^{8}$ In this regard, we found a higher rate of ICU admission in the oldest group than in the older group and determined that age $\geq 80$ years was an independent risk factor for ICU admission.

Regarding the aetiology of AP, the most common cause of AP is biliary in both ageing and younger patients. However, biliary aetiology affects a much higher percentage of ageing patients than younger AP patients. In this study, we found that the proportion of patients with biliary pancreatitis as the aetiology of AP was not significantly different between the older and oldest groups. This result was in line with previous studies showing that a higher incidence rate of gallstone-inducing AP is related to increasing age. In addition, we found that alcohol-induced AP and HTG-induced AP were less frequently encountered in ageing patients. Previous studies have reported that alcohol-induced AP occurs more often in younger subjects. ${ }^{5} \mathrm{HTG}$, which accounts for $40 \%$ of all AP cases in China, has been declining and was responsible for a small proportion of the AP aetiology. We speculate that more frequent gallstone-induced AP among ageing patients is associated with more frequent gallstone disease and a much thicker diameter of the common bile duct, which renders ageing patients more prone to the onset of biliary-induced $\mathrm{AP}^{33,34}$ Although one study reported a more severe clinical course among patients with biliaryinduced $\mathrm{AP}^{35}{ }^{35}$ we did not discover a relationship between gallstone-induced pancreatitis and mortality, ICU admission, or LOS in the multivariate logistic analysis of our ageing AP patients. This is in line with the hypothesis that the worst clinical outcomes in ageing AP patients are mainly explained by risk factors other than aetiology. ${ }^{36}$

In terms of operative treatment, ERCP was the most common operation performed in the ageing patients in our study. A similar rate of operative treatment with ERCP was verified between the two groups, likely due to the proportion of patient with an aetiology of biliary cancer. In addition, an interesting result of our study was that 19 patients aged 60-79 years underwent cholecystectomy but that no patients aged $\geq 80$ years underwent this operation. A previous study stated that the performance of cholecystectomy during hospitalization vastly reduces the risk of AP recurrence and that undergoing surgery may be related to a high rate of infection. ${ }^{13}$ Another study reported a higher complication rate after cholecystectomy in patients aged $\geq 80$ years old with complicated biliary disease than in those with uncomplicated gallstone disease. ${ }^{37}$ Moreover, some authors have proposed that cholecystectomy in AP patients $\geq 80$ years old eventually increases the death rate. ${ }^{38}$ The discrepancy in the use of cholecystectomy for ageing patients reflects the trend toward avoiding operative treatment in cases not suitable for operation. ${ }^{16}$ In reality, the approach conforms to the situation in China in which ageing patients often refuse to undergo surgical procedures in hospitals that can alleviate the disease. They fear a poor prognosis and high rates of infection and mortality. In our analysis, none of the operative treatments influenced the mortality rate or ICU admission rate. Nonetheless, PCD and necrosectomy as operative treatments were independent risk factors for LOS in patients aged $\geq 80$ years old.

The relationship between increasing age and the death rate in AP patients was an important finding in our study. An obviously higher rate of death was observed in the oldest group than in the older group. Moreover, we found that age $\geq 80$ years old was an independent risk factor for death. The other risk factor was organ failure, which included acute respiratory failure, shock and multi-organ failure. This is in line with previous reports that increasing age is closely related to a higher mortality rate in AP. ${ }^{17-20,33,34,39}$ We predicted that the higher death rate of the ageing AP patients in the oldest group was mainly attributed to the remarkable fragility of ageing patients and to a more severe clinical course of AP because the patients in both groups presented similar rates of complications and operative treatment. Interestingly, our research found a death rate of $10.1 \%$ for all ageing patients, which is lower than that reported in other studies. A previous study reported that the death rate reached $20 \%$ in patients aged over 55 years, ${ }^{33,40}$ and a death rate of up to $25 \%$ for AP patients aged $\geq 80$ years has also been reported. ${ }^{16,40}$ In contrast, a lower death rate $(7.1 \%)$ was found in a recent study of patients over 65 years old. ${ }^{13}$ The discrepancy in the death rate between our research and other studies is associated with the early diagnosis and different proportions of disease severity.

\section{Conclusion}

Our results suggest that there is a close relationship between increasing age and the death rate and LOS in AP patients. In 
addition, the results of this study drive us to pay more attention to the clinical symptoms of this frail portion of the population. Based on our findings, we propose that more comprehensive and goal-directed attendant diagnostic procedures should be performed to detect the disease early and to improve the outcomes of ageing patients.

\section{Ethics Approval and Consent to Participate}

The study was approved by the ethics committee of the First Affiliated Hospital of Nanchang University (database approval number: 2011001) prior to data collection. All procedures followed were in accordance with the ethical standards of the responsible committee on human experimentation (institutional and national) and with the Helsinki Declaration of 1975, as revised in 2008. The ethics committee of the First Affiliated Hospital of Nanchang University approved the verbally informed consent process. We confirmed that all involved persons gave their informed consent (verbal) prior to study inclusion, and we will protect patient privacy.

\section{Acknowledgment}

We thank Fengwen Xie for helpful suggestions in data collection.

\section{Author Contributions}

All authors jointly contributed to data analysis and to drafting and critically revising the manuscript, and they agree to be held fully accountable for all aspects of the work. All authors reviewed and approved the final version of the manuscript to be published.

\section{Disclosure}

The authors report no conflicts of interest in this work.

\section{References}

1. Eurostat statistics explained. Increas in the share of the population aged 65 years or over between 2009 and 2019. Available from: https:// ec.europa.eu/eurostat/statistics-explained/index.php?title=Population structure_and_ageing. Accessed April 1, 2019.

2. Gluszek S, Koziel D. Prevalence and progression of acute pancreatitis in the Swietokrzyskie Voivodeship population. Pol Przegl Chir. 2012;84(12):618-625. doi:10.2478/v10035-012-0102-4

3. Yadav D, Lowenfels AB. Trends in the epidemiology of the first attack of acute pancreatitis: a systematic review. Pancreas. 2006;33(4):323330. doi:10.1097/01.mpa.0000236733.31617.52

4. Roberts SE, Morrison-Rees S, John A, Williams JG, Brown TH, Samuel DG. The incidence and aetiology of acute pancreatitis across Europe. Pancreatology. 2017;17(2):155-165. doi:10.1016/j.pan.2017. 01.005
5. Yadav D, Lowenfels AB. The epidemiology of pancreatitis and pancreatic cancer. Gastroenterology. 2013;144(6):1252-1261. doi:10.1053/j.gastro.2013.01.068

6. Carr RA, Rejowski BJ, Cote GA, Pitt HA, Zyromski NJ. Systematic review of hypertriglyceridemia-induced acute pancreatitis: a more virulent etiology? Pancreatology. 2016;16(4):469-476. doi:10.1016/ j.pan.2016.02.011

7. Scherer J, Singh VP, Pitchumoni CS, Yadav D. Issues in hypertriglyceridemic pancreatitis: an update. J Clin Gastroenterol. 2014;48 (3):195-203. doi:10.1097/01.mcg.0000436438.60145.5a

8. Gloor B, Ahmed Z, Uhl W, Buchler MW. Pancreatic disease in the elderly. Best Pract Res Clin Gastroenterol. 2002;16(1):159-170. doi:10.1053/bega.2002.0272

9. Neoptolemos JP, Raraty M, Finch M, Sutton R. Acute pancreatitis: the substantial human and financial costs. Gut. 1998;42(6):886-891. doi:10.1136/gut.42.6.886

10. Banks PA, Freeman ML. Practice guidelines in acute pancreatitis. $A m$ $J$ Gastroenterol. 2006;101(10):2379-2400. doi:10.1111/j.1572-0241. 2006.00856.X

11. Swaroop VS, Chari ST, Clain JE. Severe acute pancreatitis. JAMA. 2004;291(23):2865-2868. doi:10.1001/jama.291.23.2865

12. Whitcomb DC. Clinical practice. Acute pancreatitis. $N$ Engl J Med. 2006;354(20):2142-2150. doi:10.1056/NEJMcp054958

13. Quero G, Covino M, Ojetti V, et al. Acute pancreatitis in oldest old: a 10 -year retrospective analysis of patients referred to the emergency department of a large tertiary hospital. Eur J Gastroenterol Hepatol. 2020;32(2):159-165. doi:10.1097/MEG.0000000000001570

14. Gullo L, Migliori M, Olah A, et al. Acute pancreatitis in five European countries: etiology and mortality. Pancreas. 2002;24 (3):223-227. doi:10.1097/00006676-200204000-00003

15. Kim JE, Hwang JH, Lee SH, et al. The clinical outcome of elderly patients with acute pancreatitis is not different in spite of the different etiologies and severity. Arch Gerontol Geriatr. 2012;54(1):256-260. doi:10.1016/j.archger.2011.01.004

16. Somasekar K, Foulkes R, Morris-Stiff G, Hassn A. Acute pancreatitis in the elderly - Can we perform better? Surgeon. 2011;9(6):305-308. doi:10.1016/j.surge.2010.11.001

17. Xin MJ, Chen H, Luo B, Sun JB. Severe acute pancreatitis in the elderly: etiology and clinical characteristics. World $J$ Gastroenterol. 2008;14(16):2517-2521. doi:10.3748/wjg.14.2517

18. de Beaux AC, Palmer KR, Carter DC. Factors influencing morbidity and mortality in acute pancreatitis; an analysis of 279 cases. Gut. 1995;37(1):121-126. doi:10.1136/gut.37.1.121

19. Gardner TB, Vege SS, Chari ST, et al. The effect of age on hospital outcomes in severe acute pancreatitis. Pancreatology. 2008;8(3):265270. doi:10.1159/000134274

20. Miki C, Kusunoki M, Inoue Y, et al. Remodeling of the immunoinflammatory network system in elderly cancer patients: implications of inflamm-aging and tumor-specific hyperinflammation. Surg Today. 2008;38(10):873-878. doi:10.1007/s00595-008-3766-y

21. Quero G, Covino M, Fiorillo C, et al. Acute pancreatitis in elderly patients: a single-center retrospective evaluation of clinical outcomes. Scand J Gastroenterol. 2019;54(4):492-498. doi:10.1080/00365 521.2019.1588369

22. Banks PA, Bollen TL, Dervenis C, et al. Classification of acute pancreatitis-2012: revision of the Atlanta classification and definitions by international consensus. Gut. 2013;62(1):102-111. doi:10.1136/gutjnl-2012-302779

23. Li W, Zhang L, Li J, et al. [Determinant-based classification of acute pancreatitis severity: an international multidisciplinary consultation: the 2013 Chinese edition]. Zhonghua Wai Ke Za Zhi. 2014;52 (5):321-324.

24. Schepers NJ, Bakker OJ, Besselink MG, et al. Early biliary decompression versus conservative treatment in acute biliary pancreatitis (APEC trial): study protocol for a randomized controlled trial. Trials. 2016;17:5. doi:10.1186/s13063-015-1132-0 
25. Sandzen B, Rosenmuller M, Haapamaki MM, et al. First attack of acute pancreatitis in Sweden 1988-2003: incidence, aetiological classification, procedures and mortality - a register study. $B M C$ Gastroenterol. 2009;9:18. doi:10.1186/1471-230X-9-18

26. Fu S, Stanek A, Mueller CM, et al. Acute pancreatitis in aging animals: loss of pancreatitis-associated protein protection? World J Gastroenterol. 2012;18(26):3379-3388. doi:10.3748/wjg. v18.i26.3379

27. Coelho A, Machado M, Sampietre SN, et al. Local and systemic effects of aging on acute pancreatitis. Pancreatology. 2019;19 (5):638-645. doi:10.1016/j.pan.2019.06.005

28. Covino M, Petruzziello C, Onder G, et al. A 12-year retrospective analysis of differences between elderly and oldest old patients referred to the emergency department of a large tertiary hospital. Maturitas. 2019;120:7-11. doi:10.1016/j.maturitas.2018.11.011

29. Murayama H, Liang J, Shaw BA, et al. Age and gender differences in the association between body mass index and all-cause mortality among older Japanese. Ethn Health. 2018;1-14.

30. Tamakoshi A, Yatsuya H, Lin Y, et al. BMI and all-cause mortality among Japanese older adults: findings from the Japan collaborative cohort study. Obesity (Silver Spring). 2010;18(2):362-369. doi:10.1038/oby.2009.190

31. Sahyoun NR, Jacques PF, Dallal G, et al. Use of albumin as a predictor of mortality in community dwelling and institutionalized elderly populations. $J$ Clin Epidemiol. 1996;49(9):981-988. doi:10.1016/0895-4356(96)00135-7

32. Closa D, Sabater L, Fernandez-Cruz L, et al. Activation of alveolar macrophages in lung injury associated with experimental acute pancreatitis is mediated by the liver. Ann Surg. 1999;229(2):230-236. doi:10.1097/00000658-199902000-00011
33. Malik AM. Biliary pancreatitis. Deadly threat to the elderly. Is it a real threat? Int J Health Sci (Qassim). 2015;9(1):35-39. doi:10.12 $816 / 0024681$

34. Turnbull IR, Clark AT, Stromberg PE, et al. Effects of aging on the immunopathologic response to sepsis. Crit Care Med. 2009;37 (3):1018-1023. doi:10.1097/CCM.0b013e3181968f3a

35. Zheng Y, Zhou Z, Li H, et al. A multicenter study on etiology of acute pancreatitis in Beijing during 5 years. Pancreas. 2015;44 (3):409-414. doi:10.1097/MPA.0000000000000273

36. Losurdo G, Iannone A, Principi M, et al. Acute pancreatitis in elderly patients: a retrospective evaluation at hospital admission. Eur J Intern Med. 2016;30:88-93. doi:10.1016/j.ejim.2016.01.011

37. Yu W, Hu S, Qi Y, et al. The correlation between sonographic diagnosis and laparoscopic observations on fatty liver. $J$ Laparoendosc Adv Surg Tech A. 2009;19(2):163-169. doi:10.1089/ lap.2008.0311

38. Tambyraja AL, Kumar S, Nixon SJ. Outcome of laparoscopic cholecystectomy in patients 80 years and older. World J Surg. 2004;28 (8):745-748. doi:10.1007/s00268-004-7378-4

39. Starr ME, Ueda J, Yamamoto $S$, et al. The effects of aging on pulmonary oxidative damage, protein nitration, and extracellular superoxide dismutase down-regulation during systemic inflammation. Free Radic Biol Med. 2011;50(2):371-380. doi:10.1016/j. freeradbiomed.2010.11.013

40. Koziel D, Gluszek-Osuch M, Suliga E, et al. Elderly persons with acute pancreatitis - specifics of the clinical course of the disease. Clin Interv Aging. 2019;14:33-41. doi:10.2147/CIA.S188520
Clinical Interventions in Aging

\section{Publish your work in this journal}

Clinical Interventions in Aging is an international, peer-reviewed journal focusing on evidence-based reports on the value or lack thereof of treatments intended to prevent or delay the onset of maladaptive correlates of aging in human beings. This journal is indexed on PubMed Central, MedLine, CAS, Scopus and the Elsevier

\section{Dovepress}

Bibliographic databases. The manuscript management system is completely online and includes a very quick and fair peer-review system, which is all easy to use. Visit http://www.dovepress.com/ testimonials.php to read real quotes from published authors. 